\title{
A systematic review of the magnitude and cause of geographic variation in unplanned hospital admission rates and length of stay for ambulatory care sensitive conditions
}

John Busby ${ }^{1 *}$, Sarah Purdy ${ }^{2}$ and William Hollingworth ${ }^{3}$

\begin{abstract}
Background: Unplanned hospital admissions place a large and increasing strain on healthcare budgets worldwide. Many admissions for ambulatory care sensitive conditions (ACSCS) are thought to be preventable, a belief supported by significant geographic variations in admission rates. We conducted a systematic review of the evidence on the magnitude and correlates of geographic variation in ACSC admission rates and length of stay (LOS).

Methods: We performed a search of Medline and Embase databases for English language cross-sectional and cohort studies on 28th March 2013 reporting geographic variation in admission rates or LOS for patients receiving unplanned care across at least 10 geographical units for one of 35 previously defined ACSCs. Forward and backward citation searches were undertaken on all included studies. We provide a narrative synthesis of study findings. Study quality was assessed using a modified Newcastle-Ottawa scale.

Results: We included 39 studies comprising 25 on admission rates and 14 on LOS. Studies generally compared admission rates between regions (e.g. states) and LOS between hospitals. Most of the published research was undertaken in the US, UK or Canada and often focussed on patients with pneumonia, COPD or heart failure. 35 (90\%) studies concluded that geographic variation was present. Primary care quality and secondary care access were frequently suggested as drivers of admission rate variation whilst secondary care quality and adherence to clinical guidelines were often listed as contributors to LOS variation. Several different methods were used to quantify variation, some studies listed raw data, failed to control for confounders and used naive statistical methods which limited their utility.

Conclusions: The substantial geographical variations in the admission rates and LOS of potentially avoidable conditions could be a symptom of variable quality of care and should be a concern for clinicians and policymakers. Policymakers targeting a reduction in unplanned admissions could introduce initiatives to improve primary care access and quality or develop alternatives to admission. Those attempting to curb unnecessarily long LOS could introduce care pathways or guidelines. Methodological work on the quantification and reporting of geographic variation is needed to aid inter-study comparisons.
\end{abstract}

Keywords: Geographical distribution, Ambulatory care, Patient admission/sn [Statistics \& Numerical Data], Length of stay/sn [Statistics \& Numerical Data], Primary care

\footnotetext{
* Correspondence: john.busby@bristol.ac.uk

${ }^{1}$ School of Social and Community Medicine, University of Bristol, Room 2.07,

Canynge Hall, 39 Whatley Road, Bristol BS8 2PS, UK

Full list of author information is available at the end of the article
}

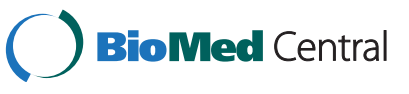

(c) 2015 Busby et al. Open Access This article is distributed under the terms of the Creative Commons Attribution 4.0 International License (http://creativecommons.org/licenses/by/4.0/, which permits unrestricted use, distribution, and reproduction in any medium, provided you give appropriate credit to the original author(s) and the source, provide a link to the Creative Commons license, and indicate if changes were made. The Creative Commons Public Domain Dedication waiver (http://creativecommons.org/publicdomain/zero/1.0/) applies to the data made available in this article, unless otherwise stated. 


\section{Background}

Unplanned admissions place a tremendous strain on healthcare resources worldwide. They account for $67 \%$ of hospital bed days at a cost of $£ 12.5 \mathrm{bn}$ per year and have risen by $47 \%$ over the last 15 years in England [1] and $13 \%$ between 2000 and 2009 in the USA [2]. Reasons for increases are manifold however demographic changes, increasing pressure on emergency department services, policy changes (e.g. emergency department waiting time targets) and evolving medical practices (e.g. increasing use of day-case surgery) are contributing factors [1]. Concerns that up to $29 \%$ of English unplanned admissions are avoidable [3] have been fuelled by the increasing proportion of short stay admissions, which could be indicative that the admission was unnecessary, and the large variations in admission rates and other process measures between healthcare units $[1,4]$. Within a context of shrinking real-terms budgets, healthcare systems worldwide have to deliver care to ageing populations with complex healthcare needs, therefore better understanding of the causes of avoidable admissions is urgently needed.

Opportunities for improved efficiency extend past the initial decision to admit; the length of time a patient spends in hospital, which will ultimately depend on the appropriateness of measures taken to stabilise, diagnose and treat the patient, has substantial clinical and resource implications. Timely discharge is dependent on a range of factors, for example limiting the number of hospital acquired infections [5], but is also heavily reliant on close integration between the hospital and other parts of the health and social care system [6-8]. In the UK, 830,000 bed days were lost in 2013 due to delayed discharge despite patients being medically fit to leave hospital, a figure which represents a rise of $9 \%$ on the previous year [1].

Recent research has focused on reducing admissions due to ambulatory care sensitive conditions (ACSCs) as a means to improve efficiency [9-11]. ACSCs are a subset of diseases where hospital admission is potentially avoidable by preventing the onset of disease (e.g. influenza vaccination), controlling an acute episodic illness (e.g. dehydration and gastroenteritis), or managing a chronic condition effectively (e.g. complications of diabetes) [12].

Observational evidence on the geographic variation in ACSC care could indicate clinical topics where interventions to improve care pathways are most needed and identify regions of the country where quality of care may be poor and further investigation is required. Interpretation of geographic variation is not straightforward and varies by the type of care being studied. Previous work has identified three distinct care types [13]: 1) Effective care where there is strong evidence of efficacy, all well-informed patients would want treatment (e.g. thrombolysis after ischaemic stroke). Geographic variations are likely to reflect underuse in low rate regions. 2) Preference sensitive care where a trade-off between treatments with different risks and benefits is required (e.g. lumpectomy or mastectomy breast cancer treatment). In this situation the appropriate rate could be driven entirely by patient preferences however this is rarely the case, as physician preferences or local practice patterns often play an important role, meaning the optimal rate is unknown and geographic variations are difficult to interpret. 3) Supply sensitive care where there are no consensus on the optimal rate of treatment and rates are largely driven by capacity at the local level. Hospital care for ACSCs is likely to be, at least in part, 'supply sensitive' as there is often little evidence on the optimal threshold for admission or appropriate LOS.

The aim of this study is to review the published literature of cross-sectional and cohort studies exploring the magnitude and causes of geographic variation within countries in ACSC admissions and LOS.

\section{Methods}

\section{Search strategy}

We searched all studies included within the Medline (published since 1950) and Embase (published since 1980) databases on 28th March 2013. We used MESH and textword terms for unplanned hospital admission or LOS, geographic variations and observational study designs to identify studies of potential interest. Forward and backward citation searches were undertaken on all studies included from the electronic search using the Web of Science. Full details of the electronic search strategy are given in Additional file 1: Appendix 1.

\section{Eligibility criteria}

Studies were eligible if they used cross-sectional or cohort study designs, were published in English and reported geographic variation in unplanned hospital admission rates or LOS for patients with an ACSC. The list of included ACSCs was based on a systematic review [14] and is provided in Additional file 1: Appendix 2. We included studies which provided any summary measure of geographic variation (e.g. range, coefficient of variation) or studies that provided raw data (e.g. regional rates displayed in tables or plotted on graphs) without any quantitative summary measure. We excluded studies with less than ten geographical units as they were too small to estimate robust measures of variation or identify the drivers of admission rates or LOS. Studies describing admission to the intensive care or emergency department attendance, reporting crude admission counts without accounting for differences in the size of region populations and those set outside OECD countries were also excluded from the analysis. All titles and abstracts were screened for inclusion by one reviewer (JB) and if deemed potentially relevant, full text articles were retrieved. A sample of 
titles and abstracts, including all those initially selected for full text review and a random sample of those initially excluded, were independently checked by a second reviewer (CC) to assess the reliability of screening.

\section{Data extraction and quality assessment}

One reviewer (JB) extracted data using a standard form. Twenty percent of these were checked by a second reviewer (WH or SP). Data were extracted on the number of admissions, characteristics of patients, number and type of geographical units, statistical methods, results and authors conclusions on the magnitude and causes of variation. Study quality was assessed by the same reviewer (JB) using a modified Newcastle Ottawa Scale for cohort [15] and cross-sectional studies [16].

\section{Data analysis}

We compared the title and abstract screening interrater reliability using the kappa statistic [17] and percentage agreement and described the characteristics of the studies included in the review. We analysed studies evaluating admission rates separately from those reporting LOS. The study results were not pooled due to heterogeneous patient populations and statistical methods used to summarise variation. Therefore we carried out a narrative synthesis of the study results. We characterised geographic units as organisational if patients were grouped according to membership or admission to an organisation (e.g. hospital, GP practice) and geographical if patients were grouped into geographical boundaries (e.g. states, primary care trusts). We reported the authors conclusions on the magnitude of variation and separated these into 4 groups (significant variation, variation exists, insignificant variation and no conclusions) based on the strength of the language used. We extracted the causes of variation proposed by the authors, for those tested empirically we extracted the variables tested, for those were causes were hypothesised in the manuscript we extracted the key phrases related to the cause.

\section{Results}

Search

Of the 5,217 non-duplicate studies retrieved through the electronic search 59 were included after title and abstract screening. A sample of 300 (5.7\%) studies of the 5,217 found during the initial electronic search were checked by a second reviewer; percentage agreement was $97 \%$ and kappa value 0.908 indicating excellent agreement [18].

Thirty-one studies were excluded during full text screening leaving 28 studies from the initial electronic search (Fig. 1). The most common causes for exclusion were a failure to report data on admission rates or LOS $(\mathrm{n}=16 ; 51.6 \%)$, the study contained less than 10 geographical units $(\mathrm{n}=7 ; 22.6 \%)$ or the study did not report geographic variation $(n=4 ; 12.9 \%)$. Citation searches of the included studies yielded a further 1,076 titles and abstracts for screening, 8 of which were included. A further 3 eligible studies were found during a separate unpublished systematic review on readmission rates. In total, the review comprised 39 primary studies. There were no substantive differences between reviewers on the $20 \%$ sample of papers there were double extracted, hence the remaining $80 \%$ of studies were extracted by a single reviewer (JB).

\section{Study quality}

A summary of the study quality assessment for the cross-sectional studies investigating geographic variation in admission rates is given in Additional file 1: Appendix 3 and Appendix 4. Studies scored well on the group selection domain as they generally included large sample sizes that were highly representative of patients in the wider community. Scores on the comparability domain were mixed due to a failure to adjust for potentially important case-mix differences (i.e. age and sex) between units. All studies assessed outcomes using medical records which were considered to be at low risk of bias and, as follow-up was only required until discharge for LOS studies, they were generally deemed to be adequate in the outcome domain although the use of naïve statistical methods, for example reporting results using the range, lowered this score in some cases.

\section{Study characteristics}

Twenty-five (64\%) of the included studies reported data on admission rates (Table 1$)$. Five studies $(20 \%)$ did not report how many admissions were included, of those that did most were large; 15 of 20 (75\%) were comprised of more than 10,000 patients. Studies varied in both the number (range: 10-3,187) and type of geographic units studied; in 25 of 27 comparisons (93\%) were between geographical units (e.g. counties, states). Studies were set in 5 different countries, with the USA ( $n=10$; $40 \%$ ) commonest, and included several different ACSCs; pneumonia ( $n=6 ; 24 \%$ ) was most frequently analysed.

Fourteen studies (36 \%) reported data on LOS (Table 2) and were typically smaller than those for admission rates; seven (50\%) included less than 5,000 patients. Studies varied in the number of units compared (range: $10-3,135)$; the vast majority $(n=13 ; 93 \%)$ investigated differences between hospitals. Studies were set in six different countries, most commonly the UK $(n=4 ; 29 \%)$, USA $(\mathrm{n}=3 ; 29 \%)$ and Spain $(\mathrm{n}=3 ; 21 \%)$, while patients with pneumonia $(n=6 ; 3 \%)$ or COPD $(n=4 ; 29 \%)$ were most frequently analysed. 


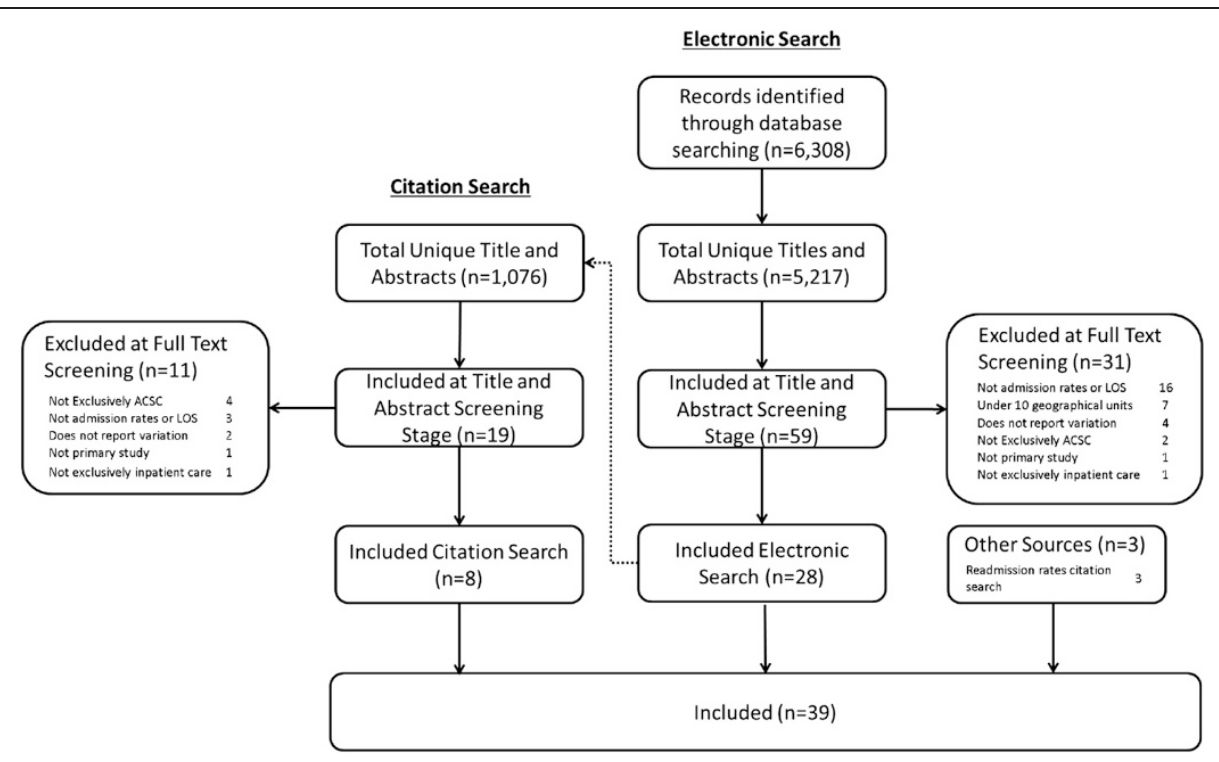

Fig 1 Study flow diagram

\section{Reporting methods and covariate adjustment}

Twenty-two (88 \%) of the admission rate studies reported raw data using maps $(\mathrm{n}=14 ; 64 \%)$, graphs $(\mathrm{n}=5 ; 23 \%)$ or tables $(\mathrm{n}=5 ; 23 \%)$. Twelve studies $(48 \%)$ estimated a summary measure for variation. Most frequently this was with spatial analysis $(\mathrm{n}=7 ; 58 \%)$, such as Morans $\mathrm{I}^{2}$, while two studies each reported the coefficient of variation and the systematic component of variation (17\%). Most studies ( $\mathrm{n}=19 ; 76 \%$ ) adjusted their analysis for covariates that might affect the clinical need for hospital admission, 15 (60\%) studies adjusted for the age and sex of the population, seven of these (28\%) additionally adjusted for casemix differences or other factors (e.g. deprivation, ethnicity).

Most ( $\mathrm{n}=9 ; 64 \%)$ LOS studies reported raw data using graphs $(n=6 ; 67 \%)$ or tables $(n=3 ; 33 \%)$. Twelve studies (86\%) estimated a summary measure for variation. Of these the majority were crude measures (e.g. IQR). Only 2 used methods (e.g. hierarchical model variance) that distinguish systematic from random variation. In contrast to admission rate analyses, adjustment for covariates was undertaken in only half of studies $(n=7)$, five (36\%) adjusted for age, sex and case-mix while one other $(7 \%)$ additionally controlled for other factors (i.e. alcohol consumption, blood culture results).

\section{Magnitude of variation}

Two (8\%) studies did not offer conclusions on the magnitude of admission rate variation. Of the remainder 21 (91 \%) concluded that some geographic variation was present of which 16 (64\%) reported significant variation (Table 3). For example, one high-quality US study including 55 million heart failure admissions concluded that "Risk-standardized HF hospitalization varied significantly by state" with admission rates ranging from 1,149 to 2,931 per 100,000 person-years [19]. One of the two exceptions which reported no significant variation was based in the UK and found minimal differences in unplanned admission rates for several ACSCs between 94 GP practices after adjustment for age, sex and deprivation differences [20].

All 13 studies which commented on geographic variation in LOS concluded it existed. Ten (77 \%) reported significant variation (Table 4), for example, a high quality Danish study of 11,332 pneumonia admissions at 16 hospitals concluded significant regional differences in median LOS, ranging from two to seven days, were present [21].

\section{Causes of variation}

Several reasons for the substantial admission rate variation were proposed (Table 1$)$. Most studies $(n=22 ; 88 \%)$ noted that unmeasured case-mix differences could be present despite, in many cases, their best efforts at adjustment. Ease of secondary care access $(\mathrm{n}=11 ; 44 \%)$ and inadequate primary care quality $(n=5 ; 20 \%)$ or access $(n=4 ; 16 \%)$ were often cited as key drivers of admission rate variation while several studies $(n=6 ; 24 \%)$ noted that coding quality differences between units could have led to some spurious variation.

Some studies went further and empirically tested the effect of potentially important factors on unplanned admission rates. Increased secondary care access, and in particular bed availability, was reported to be associated with higher admission rates in three studies spanning a wide range of conditions (COPD, asthma, gastroenteritis and diabetes) [22-24] although another study on pneumonia admissions reported no association [25]. Two studies investigated the effect of hospital staffing levels on 
Table 1 Study details, admission rates

\begin{tabular}{|c|c|c|c|c|c|c|c|c|}
\hline Paper ID & Condition & Number of Admissions & Geographical Units $(\mathrm{N})^{a}$ & Mean Age $(S D)^{b}$ & $\begin{array}{l}\text { Covariate } \\
\text { Adjustment }^{c}\end{array}$ & Statistical Methods & Tested Cause & Untested Cause \\
\hline \multicolumn{9}{|l|}{ Australia } \\
\hline \multirow[t]{5}{*}{ Ansari 2005 [56] } & Diabetes & 38,900 & Primary Care Partnerhips (32) & N/A & $\mathrm{A}, \mathrm{S}$ & Raw Data & None & Case Mix \\
\hline & & & & & & Graphs & & SC Access \\
\hline & & & & & & & & SC Quality \\
\hline & & & & & & & & Clinical Guidelines \\
\hline & & & & & & & & Coding Quality \\
\hline \multirow[t]{2}{*}{ Tennant 2000 [57] } & Dental & 3,754 & Health service region (32) & $<18(100 \%)$ & $A$ & Raw Data & None & None \\
\hline & & & & & & Graphs & & \\
\hline \multicolumn{9}{|l|}{ Canada } \\
\hline \multirow[t]{5}{*}{ Crighton 2007 [58] } & Influenza & 241,803 & County (49) & N/A & $\mathrm{A}, \mathrm{S}$ & Raw Data & None & Case Mix \\
\hline & Pneumonia & & & & & Maps & & SC Access \\
\hline & & & & & & Spatial Analysis & & PC Quality \\
\hline & & & & & & Range Analysis & & Clinical Guidelines \\
\hline & & & & & & COV & & \\
\hline \multirow[t]{3}{*}{ Crighton 2008 [59] } & Influenza & 241,803 & County (49) & N/A & $\mathrm{A}, \mathrm{S}$ & Raw Data & None & Case Mix \\
\hline & Pneumonia & & & & & Maps & & Coding Quality \\
\hline & & & & & & Spatial Analysis & & \\
\hline \multirow[t]{3}{*}{ Curtis 2002 [60] } & Diabetes & 15,872 & District Health Board (16) & $<18(100 \%)$ & $\mathrm{A}, \mathrm{S}$ & Raw Data & None & Case Mix \\
\hline & & & & & & Maps & & PC Quality \\
\hline & & & & & & Extremal Quotient & & \\
\hline \multirow[t]{5}{*}{ Jin 2003 [25] } & Pneumonia & 36,516 & Health Region (17) & $18-44(18 \%)$ & $\mathrm{A}, \mathrm{S}$ & Raw Data & SC Access & Case Mix \\
\hline & & & & 46-64 (19 \%) & & Graphs & & \\
\hline & & & & $65-74(20 \%)$ & & & & \\
\hline & & & & $75-84(26 \%)$ & & & & \\
\hline & & & & $85+(16 \%)$ & & & & \\
\hline \multirow[t]{8}{*}{ To 1996 [24] } & Gastroenteritis & 10,105 & County (41) & $<1(25.3 \%)$ & $\mathrm{A}, \mathrm{S}$ & Range & Case Mix & Coding Quality \\
\hline & & & & $1(25.7 \%)$ & & COV & SC Access & \\
\hline & & & & $2(14.5 \%)$ & & SCV & & \\
\hline & & & & 3-5 (17.3\%) & & Extremal Quotient & & \\
\hline & & & & $6-8(7.0 \%)$ & & & & \\
\hline & & & & 9-14 (4.4\%) & & & & \\
\hline & & & & $12-14(2.9 \%)$ & & & & \\
\hline & & & & $15-17$ (2.8 \%) & & & & \\
\hline
\end{tabular}


Table 1 Study details, admission rates (Continued)

\begin{tabular}{|c|c|c|c|c|c|c|c|c|}
\hline \multicolumn{9}{|l|}{ New Zealand } \\
\hline \multirow[t]{2}{*}{ Bandaranayake 2011 [61] } & Influenza & 1,743 & District Health Board (20) & N/A & None & Raw Data & None & Case Mix \\
\hline & & & & & & Graphs & & \\
\hline \multirow[t]{4}{*}{ Barnett 2010 [26] } & ACSCS & 24,894 & GP Practice (102) & N/A & None & Raw Data & Case Mix & None \\
\hline & & & & & & Graphs & PC Quality & \\
\hline & & & & & & & PC Access & \\
\hline & & & & & & & Practice Size & \\
\hline \multirow[t]{2}{*}{ Dharmalingam 2004 [62] } & ACSCS & N/A & Modified District Health & N/A & A & Raw Data & Case Mix & None \\
\hline & & & & & & Tables & & \\
\hline \multirow[t]{2}{*}{ Ellison-Loschmann 2004 [53] } & Asthma & 25,865 & Territorial Authority (74) & N/A & None & Raw Data & None & Case Mix \\
\hline & & & & & & Maps & & \\
\hline \multicolumn{9}{|l|}{ Spain } \\
\hline \multirow[t]{6}{*}{ Magan 2008 [63] } & ACSCS & 64,409 & Health District (34) & 78.9 & $\mathrm{~A}, \mathrm{~S}$ & Raw Data & Case Mix & PC Quality \\
\hline & Cardiovascular Disease & & & & & Maps & & Clinical Guidelines \\
\hline & Heart Failure & & & & & Tables & & Staffing Levels \\
\hline & Pneumonia & & & & & Range & & \\
\hline & & & & & & COV & & \\
\hline & & & & & & SCV & & \\
\hline \multicolumn{9}{|l|}{ UK } \\
\hline \multirow[t]{5}{*}{ Downing 2007 [20] } & Asthma & 2,271 & GP Practice (94) & $<65(84.8 \%)$ & $\mathrm{A}, \mathrm{S}, \mathrm{O}$ & Hierarchical Model & Case Mix & None \\
\hline & Cardiovascular Disease & & & $>65(15.2 \%)$ & & Variance Estimates & PC Quality & \\
\hline & COPD & & & & & & & \\
\hline & Diabetes & & & & & & & \\
\hline & Stroke & & & & & & & \\
\hline \multirow[t]{3}{*}{ Giuffrida 1999 [22] } & Asthma & N/A & Health Authority (90) & N/A & None & Range & Case Mix & Clinical Guidelines \\
\hline & Diabetes & & & & & & SC Access & \\
\hline & & & & & & & Staffing Levels & \\
\hline \multirow[t]{2}{*}{ Starr 1996 [56] } & Stroke & N/A & Local government districts (22) & 40-59 (100 \%) & None & Raw Data & Case Mix & SC Access \\
\hline & & & & & & Tables & & \\
\hline \multicolumn{9}{|l|}{ US } \\
\hline \multirow[t]{2}{*}{ Adams 1993 [64] } & Alcohol Abuse & 87,147 & State (50) & $>65(100 \%)$ & $\mathrm{A}, \mathrm{S}, \mathrm{O}$ & Raw Data & Case Mix & Coding Quality \\
\hline & & & & & & Maps & & \\
\hline \multirow[t]{2}{*}{ Casper 2010 [65] } & Heart Failure & N/A & County $(3,187)$ & $>65(100 \%)$ & A & Raw Data & None & Coding Quality \\
\hline & & & & & & Maps & & PC Access \\
\hline
\end{tabular}


Table 1 Study details, admission rates (Continued)

\begin{tabular}{|c|c|c|c|c|c|c|c|c|}
\hline \multirow[t]{2}{*}{ Chen 2011 [19] } & Heart Failure & $55,097,390$ & State (52) & $79.0(7.7)$ & $\mathrm{A}, \mathrm{S}, \mathrm{C}, \mathrm{O}$ & Raw Data & None & None \\
\hline & & & & & & Maps & & \\
\hline \multirow[t]{2}{*}{ Gorton 2006 [66] } & Pneumonia & 4,948 & County (67) & $59.6 \mathrm{mo}$ & $\mathrm{A}, \mathrm{S}, \mathrm{O}$ & Raw Data & None & Case Mix \\
\hline & & & & & & Maps & & SC Access \\
\hline \multirow[t]{3}{*}{ Holt 2011 [67] } & COPD & $3,786,908$ & State (50) & $>65(100 \%)$ & None & Raw Data & None & Case Mix \\
\hline & & & Hospital Referral Region (949) & & & Maps & & \\
\hline & & & & & & Spatial Analysis & & \\
\hline \multirow[t]{2}{*}{ Laditka 1999 [68] } & ACSCS & 21,923 & Hospital Market Area (24) & $>65(100 \%)$ & $\mathrm{A}, \mathrm{S}$ & Raw Data & None & Case Mix \\
\hline & & & & & & Tables & & PC Access \\
\hline \multirow[t]{7}{*}{ Lanska 1994 [69] } & Stroke & 318,000 & State (49) & $>65(100 \%)$ & $\mathrm{A}, \mathrm{S}, \mathrm{O}$ & Raw Data & None & Case Mix \\
\hline & & & & & & Maps & & SC Access \\
\hline & & & & & & Spatial Analysis & & SC Access \\
\hline & & & & & & & & Clinical Guidelines \\
\hline & & & & & & & & Procedure/Drug \\
\hline & & & & & & & & Availability \\
\hline & & & & & & & & High readmission rates \\
\hline \multirow[t]{3}{*}{ Maliszewski 2011 [60] } & Influenza & 2,010 & County (58) & $<18(24.4 \%)$ & $\mathrm{A}, \mathrm{S}, \mathrm{O}$ & Raw Data & Case Mix & None \\
\hline & & & & $>65$ (12.4\%) & & Maps & & \\
\hline & & & & & & Spatial Analysis & & \\
\hline \multirow[t]{3}{*}{ Morris 1994 [23] } & Asthma & N/A & County $(3,079)$ & $>65(100 \%)$ & $\mathrm{A}, \mathrm{S}, \mathrm{O}$ & Raw Data & Case Mix & Coding Quality \\
\hline & COPD & & & & & Maps & SC Access & \\
\hline & Pneumonia & & & & & Spatial Analysis & Staffing Levels & \\
\hline \multirow[t]{4}{*}{ Ogunniyi 2012 [70] } & Heart Failure & 845,421 & County $(1,014)$ & 65-75 (30.8 \%) & A & Raw Data & None & Case Mix \\
\hline & & & State (10) & $75-84(41.3 \%)$ & & Tables & & SC Access \\
\hline & & & & $>85$ (27.9\%) & & Maps & & PC Access \\
\hline & & & & & & Spatial Analysis & & \\
\hline
\end{tabular}

${ }^{\mathrm{a}}$ Number of geographical units

${ }^{\mathrm{b}}$ Mean age and standard deviation when available. Other counts represent percentage of patients in each age band

'A: Age, S: Sex, C: Case-Mix, O: Other

dPrimary Care

eSecondary Care 
Table 2 Study details, length of stay

\begin{tabular}{|c|c|c|c|c|c|c|c|c|}
\hline Paper ID & Condition & $\begin{array}{l}\text { Number of } \\
\text { Admissions }\end{array}$ & $\begin{array}{l}\text { Geographical } \\
\text { Units }(\mathrm{N})^{\mathrm{a}}\end{array}$ & $\begin{array}{l}\text { Mean Age } \\
(S D)^{b}\end{array}$ & $\begin{array}{l}\text { Covariate } \\
\text { Adjustment }^{c}\end{array}$ & Statistical Methods & Tested Cause & Untested Cause \\
\hline \multicolumn{9}{|l|}{ Belgium } \\
\hline \multirow[t]{2}{*}{ Claeys 2013 [55] } & \multirow[t]{2}{*}{$\mathrm{Ml}$} & \multirow[t]{2}{*}{2,079} & \multirow[t]{2}{*}{ Hospital (33) } & \multirow[t]{2}{*}{$62(13)$} & \multirow[t]{2}{*}{ None } & Raw Data & \multirow[t]{2}{*}{ Case Mix } & \multirow[t]{2}{*}{ Discharge Planning } \\
\hline & & & & & & Graphs & & \\
\hline \multicolumn{9}{|l|}{ Canada } \\
\hline \multirow[t]{3}{*}{ Feagan 2000 [71] } & \multirow[t]{3}{*}{ Pneumonia } & \multirow[t]{3}{*}{858} & \multirow[t]{3}{*}{ Hospital (20) } & \multirow[t]{3}{*}{$69.4(17.7)$} & \multirow[t]{3}{*}{$\mathrm{A}, \mathrm{S}, \mathrm{C}, \mathrm{O}$} & Raw Data & Case Mix & Clinical Guidelines \\
\hline & & & & & & Tables & \multirow[t]{2}{*}{ Hospital Type } & PC Access \\
\hline & & & & & & $\%$ Variation Explained & & $\begin{array}{l}\text { Procedure/Drug } \\
\text { Availability }\end{array}$ \\
\hline \multicolumn{9}{|l|}{ Denmark } \\
\hline \multirow[t]{3}{*}{ Klausen 2012 [21] } & \multirow[t]{3}{*}{ Pneumonia } & \multirow[t]{3}{*}{12,753} & \multirow[t]{3}{*}{ Hospital (22) } & 65-74 (32.5 \%) & \multirow[t]{3}{*}{$A, S, C$} & Raw Data & Case Mix & Clinical Guidelines \\
\hline & & & & $75-84$ (40.6 \%) & & Graphs & Hospital Size & \multirow[t]{2}{*}{ PC Quality } \\
\hline & & & & $>85(26.9 \%)$ & & P-Values (Cox Regression) & Condition Volume & \\
\hline \multicolumn{9}{|l|}{ Spain } \\
\hline \multirow[t]{4}{*}{ Cabre 2004 [72] } & \multirow[t]{4}{*}{ Pneumonia } & \multirow[t]{4}{*}{1,769} & \multirow[t]{4}{*}{ Hospital (27) } & $66.4(18.1)$ & $A, S, C$ & Hierarchical Model Variance & Case Mix & SC Access \\
\hline & & & & & & Estimates & & SC Quality \\
\hline & & & & & & & & Clinical Guidelines \\
\hline & & & & & & & & PC Quality \\
\hline Garau 2008 [73] & Pneumonia & 3,233 & Hospital (10) & $66(18.5)$ & $\mathrm{A}, \mathrm{SC}, \mathrm{O}$ & Raw Data & Case Mix & None \\
\hline & & & & & & Tables & & \\
\hline & & & & & & P-Values (Cox Regression) & & \\
\hline $\begin{array}{l}\text { Pozo-Rodriguez } \\
2012 \text { [74] }\end{array}$ & COPD & 5,178 & Hospital (129) & 75 (IQR: 68-80) & None & IQR & None & None \\
\hline UK & & & & & & & & \\
\hline Hosker 2007 [75] & COPD & 8,013 & Hospital (233) & 71 (IQR: 71-74) & None & IQR & None & None \\
\hline Price 2006 [27] & COPD & 910 & Hospital (234) & N/A & $A, S, C$ & IQR & SC Quality & None \\
\hline & & & & & & ICC & Clinical Guidelines & \\
\hline & & & & & & & Hospital Size & \\
\hline Roberts 2002 [76] & COPD & 1,400 & Hospital (38) & 72 & None & Range & Case Mix & SC Quality \\
\hline & & & & & & IQR & & \\
\hline Rudd 2001 [77] & Stroke & 6,894 & Health Region (10) & $75(12)$ & $\mathrm{A}, \mathrm{O}$ & Raw Data & Case Mix & None \\
\hline & & & & & & Tables & SC Quality & \\
\hline & & & & & & P-Values (Kruskal-Wallis) & & \\
\hline
\end{tabular}


Table 2 Study details, length of stay (Continued)

\begin{tabular}{|c|c|c|c|c|c|c|c|c|}
\hline \multicolumn{9}{|l|}{$\overline{\text { US }}$} \\
\hline \multirow[t]{3}{*}{ Brogan 2012 [78] } & \multirow[t]{3}{*}{ Pneumonia } & \multirow[t]{3}{*}{43,819} & \multirow[t]{3}{*}{ Hospital (29) } & \multirow[t]{3}{*}{3 (IQR: 1-6) } & \multirow[t]{3}{*}{ None } & Raw Data & \multirow{3}{*}{$\begin{array}{l}\text { Procedure/Drug } \\
\text { Availability }\end{array}$} & \multirow[t]{3}{*}{ None } \\
\hline & & & & & & Graphs & & \\
\hline & & & & & & Range & & \\
\hline \multirow[t]{5}{*}{ Conway 2009 [28] } & \multirow[t]{5}{*}{ UTI } & \multirow[t]{5}{*}{20,892} & \multirow[t]{5}{*}{ Hospital (25) } & $1-2 \mathrm{mo}(16.7 \%)$ & \multirow[t]{5}{*}{ None } & Raw Data & Case Mix & \multirow[t]{5}{*}{ Coding Quality } \\
\hline & & & & 2-6 mo (29.9\%) & & Graphs & Clinical Guidelines & \\
\hline & & & & 6-24 mo & & & Condition Volume & \\
\hline & & & & (19.1\%) & & & & \\
\hline & & & & $2-12$ y (34.3 \%) & & & & \\
\hline \multirow[t]{3}{*}{ Drye 2012 [79] } & Heart Failure & \multirow[t]{3}{*}{718,508} & \multirow[t]{3}{*}{ Hospital $(3,135)$} & $>65$ (100 \%) & \multirow[t]{3}{*}{ None } & Raw Data & None & \multirow[t]{3}{*}{ None } \\
\hline & Ml & & & & & Graphs & & \\
\hline & Pneumonia & & & & & Range & & \\
\hline \multirow[t]{3}{*}{ Krumholz 1999 [80] } & \multirow[t]{3}{*}{ Heart Failure } & \multirow[t]{3}{*}{905} & \multirow[t]{3}{*}{ Hospital (49) } & $<65(42 \%)$ & \multirow[t]{3}{*}{$\mathrm{A}, \mathrm{S}, \mathrm{C}, \mathrm{O}$} & Raw Data & Case Mix & \multirow[t]{3}{*}{ SC Quality } \\
\hline & & & & $>65(58 \%)$ & & Graphs & & \\
\hline & & & & & & \% Variation Explained & & \\
\hline
\end{tabular}

Number of geographical units

${ }^{\mathrm{b}}$ Mean age and standard deviation when available. Other counts represent percentage of patients in each age band

'A: Age, S: Sex, C: Case-Mix, O: Other

dPrimary Care

'Secondary Care 
Table 3 Authors conclusions on variation, admission rates

\begin{tabular}{|c|c|}
\hline Paper ID & Author Conclusions \\
\hline \multicolumn{2}{|l|}{ Significant Variation } \\
\hline \multicolumn{2}{|l|}{ Australia } \\
\hline Ansari 2005 & $\begin{array}{l}\text { "There was a wide variation (almost fivefold) } \\
\text { in admission rates" }\end{array}$ \\
\hline Tennant 2000 & $\begin{array}{l}\text { "[8 of } 32 \text { regions] had significantly less } \\
\text { episodes of hospitalization....than the } \\
\text { State average" }\end{array}$ \\
\hline \multicolumn{2}{|l|}{ Canada } \\
\hline Crighton 2007 & $\begin{array}{l}\text { "Marked differences in rates between } \\
\text { counties... large variability in county } \\
\text { rates" }\end{array}$ \\
\hline Crighton 2008 & $\begin{array}{l}\text { "The heterogeneity in...hospitalization } \\
\text { rates and significant spatial clustering" }\end{array}$ \\
\hline \multicolumn{2}{|l|}{ New Zealand } \\
\hline Barnett 2010 & "Substantial variation in admission rates" \\
\hline Dharmalingam 2004 & $\begin{array}{l}\text { "Substantial geographical variation in } \\
\text { the level of avoidable hospitalisation" }\end{array}$ \\
\hline \multicolumn{2}{|l|}{ Spain } \\
\hline Magan 2008 & "Considerable variability in these rates" \\
\hline \multicolumn{2}{|l|}{ UK } \\
\hline Giuffrida 1999 & $\begin{array}{l}\text { "Clear variation....in crude admission } \\
\text { rates" }\end{array}$ \\
\hline Starr 1996 & $\begin{array}{l}\text { "There was considerable variation... } \\
\text { between districts" }\end{array}$ \\
\hline \multicolumn{2}{|l|}{ US } \\
\hline Adams 1993 & $\begin{array}{l}\text { "There was considerable geographic } \\
\text { variation" }\end{array}$ \\
\hline Casper 2010 & $\begin{array}{l}\text { "Magnitude of geographic disparity } \\
\text { was substantial between the high- and } \\
\text { low-rate counties" }\end{array}$ \\
\hline Chen 2011 & $\begin{array}{l}\text { "Rates in } 1998 \text { and } 2008 \text { varied } \\
\text { significantly by state" }\end{array}$ \\
\hline Gorton 2006 & "Rates vary widely" \\
\hline Holt 2011 & $\begin{array}{l}\text { "Substantial geographic variations in } \\
\text { COPD hospitalization risk among states } \\
\text { and HSAs" }\end{array}$ \\
\hline Laditka 1999 & $\begin{array}{l}\text { "Significant variation in preventable } \\
\text { hospitalization" }\end{array}$ \\
\hline Morris 1994 & $\begin{array}{l}\text { "The geographic distribution in hospital } \\
\text { admission rates is unequivocally } \\
\text { heterogeneous" }\end{array}$ \\
\hline \multicolumn{2}{|l|}{ Variation Exists } \\
\hline \multicolumn{2}{|l|}{ Canada } \\
\hline Curtis 2002 & $\begin{array}{l}\text { "Differences observed for DKA are } \\
\text { clinically important" }\end{array}$ \\
\hline $\operatorname{Jin} 2003$ & "The incidence of... hospitalization varies" \\
\hline To 1996 & $\begin{array}{l}\text { "Variation among the counties... was } \\
\text { moderately large" }\end{array}$ \\
\hline \multicolumn{2}{|l|}{ New Zealand } \\
\hline Bandaranayake 2011 & $\begin{array}{l}\text { "We observed a heterogeneous } \\
\text { distribution" }\end{array}$ \\
\hline
\end{tabular}

Table 3 Authors conclusions on variation, admission rates (Continued)

\begin{tabular}{|c|c|}
\hline \multicolumn{2}{|l|}{ US } \\
\hline Maliszewski 2011 & $\begin{array}{l}\text { "Hospitalization rates were dependent } \\
\text { upon neighbouring county hospitalization } \\
\text { rates" }\end{array}$ \\
\hline \multicolumn{2}{|l|}{ Insignificant Variation } \\
\hline \multicolumn{2}{|l|}{ UK } \\
\hline Downing 2007 & $\begin{array}{l}\text { "Generally the variances were small } \\
\text { meaning there was little unexplained } \\
\text { variation" }\end{array}$ \\
\hline \multicolumn{2}{|l|}{ US } \\
\hline Lanska 1994 & $\begin{array}{l}\text { "Hospitalization rates show relatively } \\
\text { little small-scale variation" }\end{array}$ \\
\hline \multicolumn{2}{|l|}{ No Conclusion } \\
\hline \multicolumn{2}{|l|}{ New Zealand } \\
\hline Ellison-Loschmann 2004 & No conclusions \\
\hline \multicolumn{2}{|l|}{ US } \\
\hline Ogunniyi 2012 & No conclusions \\
\hline
\end{tabular}

admission rates with conflicting results. The effect of primary care quality, measured using GP quality scores [20] and the number of GPs within the population [26] respectively, on admission rates was investigated in two studies with no consistent effect. One study found consistent negative associations between staffing levels and admission rates for three respiratory ACSCs [23], which they attribute to improved outpatient care, while another found positive associations for asthma, diabetes and epilepsy admissions [22].

A variety of possible drivers of LOS variation were suggested by authors (Table 2 ). In accordance with the admission rate results, nine $(64 \%)$ of the papers noted that unmeasured case mix differences between units could contribute to the observed variation. The use of clinical guidelines $(\mathrm{n}=5 ; 36 \%)$ and the quality of care received within hospital $(\mathrm{n}=5 ; 36 \%)$ were regularly cited as potentially important factors influencing LOS variation. Some studies went further and empirically investigated the factors that might be important drivers of LOS. A study on UK COPD patients found that higher quality and better organised hospitals, those with more respiratory consultants and those with an early discharge scheme or local written guidelines for follow-up had reduced odds of a length of stay $>7$ days [27]. A US study of patients with UTI [28] also reported that the presence of clinical practice guidelines is associated with shorter LOS. Three studies investigated the effect of higher condition-specific admissions or hospital size on LOS and found no or very marginal effects [28, 21, 27]. Further details on the causes of variation proposed by authors are given in Additional file 1: Appendix 5 and Appendix 6. 
Table 4 Authors conclusions on variation, length of stay

\begin{tabular}{|c|c|}
\hline Paper ID & Author Conclusions \\
\hline \multicolumn{2}{|l|}{ Significant Variation } \\
\hline \multicolumn{2}{|l|}{ Belgium } \\
\hline Claeys 2013 & "Large inter-hospital variations" \\
\hline \multicolumn{2}{|l|}{ Canada } \\
\hline Feagan 2000 & $\begin{array}{l}\text { "Considerable heterogeneity in LOS } \\
\text { was noted among the hospitals" }\end{array}$ \\
\hline \multicolumn{2}{|l|}{ Denmark } \\
\hline Klausen 2012 & $\begin{array}{l}\text { "We show significant regional } \\
\text { differences" }\end{array}$ \\
\hline \multicolumn{2}{|l|}{ Spain } \\
\hline Cabre 2004 & $\begin{array}{l}\text { "Significant variations...among the } \\
27 \text { community hospitals" }\end{array}$ \\
\hline Garau 2008 & $\begin{array}{l}\text { "Length of stay varied markedly } \\
\text { among centres" }\end{array}$ \\
\hline \multicolumn{2}{|l|}{ UK } \\
\hline Hosker 2007 & "Wide variability between hospitals" \\
\hline Price 2006 & $\begin{array}{l}\text { "The wide variation between hospital } \\
\text { units... is probably unacceptable" }\end{array}$ \\
\hline Roberts 2002 & $\begin{array}{l}\text { "The variation between hospitals... } \\
\text { was very wide" }\end{array}$ \\
\hline \multicolumn{2}{|l|}{ US } \\
\hline Conway 2009 & "We found high variability in outcomes" \\
\hline Krumholz 1999 & $\begin{array}{l}\text { "Significant inter hospital differences } \\
\text { in the unadjusted length of stay" }\end{array}$ \\
\hline \multicolumn{2}{|l|}{ Variation Exists } \\
\hline \multicolumn{2}{|l|}{ UK } \\
\hline Rudd 2001 & $\begin{array}{l}\text { "[Length of stay] varied by a mean of } \\
\text { eight days between region" }\end{array}$ \\
\hline \multicolumn{2}{|l|}{ US } \\
\hline Brogan 2012 & "LOS differed across hospitals" \\
\hline Drye 2012 & $\begin{array}{l}\text { "Mean patient LOS at the hospital level } \\
\text { varied for each condition" }\end{array}$ \\
\hline \multicolumn{2}{|l|}{ No Conclusion } \\
\hline \multicolumn{2}{|l|}{ Spain } \\
\hline Pozo-Rodriguez 2012 & No conclusions \\
\hline
\end{tabular}

\section{Discussion \\ Main findings}

Substantial geographic variation in unplanned ACSCs admission rates and LOS is commonplace in high income countries. A broad literature exists on the topic however, to date, most research has concentrated on pneumonia, heart failure and COPD and been based in the US, UK or Canada. Primary care quality and secondary care access were often cited as drivers of admission rate variation whilst secondary care quality and the use of clinical guidelines were often listed as contributors to LOS variation. Few studies went further and empirically examined the correlates of unplanned admission rates and LOS, results from those that did suggest that increased bed availability and the absence of clinical guidelines may be an important determinant of high admission rates and LOS respectively. Several different methods were used to quantify and report variation, some studies failed to control for confounders and used naive statistical methods which limited their utility.

\section{Strengths and weaknesses}

To our knowledge, this is the first study to systematically review the literature on geographical variation in unplanned ACSC admissions and LOS. Due to resource constraints only a sample of 300 titles and abstract decisions were checked by a second reviewer and $80 \%$ of the full text data extraction was undertaken by a single reviewer which could reduce accuracy. However the high inter-reviewer agreement on title and abstract screening and full-text extraction suggests that our results would not have changed markedly if double screening and extraction of all studies had been undertaken. Our electronic search strategy included only Medline and Embase databases which may have missed some important studies; however our comprehensive Web of Science citation search, which included both forward and backward citations, should limit the effect of this weakness. As many of the terms used to describe geographic variation are non-specific it was difficult to identify relevant articles which may have impaired both the sensitivity and specificity of the electronic search. We have provided a guide on the magnitude of variation present in each study by categorising the authors' conclusions as 'significant variation,' 'variation exists' and 'insignificant variation' however these classifications are subjective.

\section{Comparison with other studies}

A broad international literature exists examining variations in other facets of health care utilisation. Much of the this has built on work by Wennberg and colleagues who, since their seminal paper in 1984 [29], have developed the Dartmouth Atlas [30] in the US which has demonstrated large geographical differences in health care spending, drug use, surgical procedures and care quality over the last 30 years. Other countries have followed with their own Atlases, including England [31,32] and Spain [33], demonstrating wide levels of variation across their healthcare systems.

Despite the overwhelming evidence that significant geographic variations exist in ACSC admission rates and LOS, relatively little is known about their cause. What little evidence that does exist is in broad agreement with our findings. Secondary care access has been widely documented [13, 34] as an important factor affecting unplanned admission rates. Evidence investigating the link between primary care quality and unplanned admission 
rates is mixed; two UK based studies found small and inconsistent effects [35, 36] while another found a strong association between high quality and lower cardiovascular admission rates [37]. A recent study also reported substantially decreased admission rates for ACSC conditions where GPs had financial incentives aimed at improving the quality of care [38]. Several studies have demonstrated that clinical guidelines [39] and pathways are effective modifiers of LOS.[40, 41] The effect of secondary care quality on LOS is less clear cut, while some quality improvement initiatives, such as reducing hospital acquired infections, [42] will undoubtedly lead to shorter LOS in some cases higher quality care may necessitate longer LOS [43] leading some to argue that an 'inverted U' association between quality and LOS is likely [44], Although issues at the interface between secondary and community care has been highlighted as an important contributor to LOS variation [6-8], few of the studies in our review acknowledged this as an important issue or attempted to measure any association.

\section{Implications}

The substantial geographical variations displayed among the majority of studies in this review should be of concern for policymakers. The large differences, often after adjustment for a range of potential confounders, could be a marker of variable quality care and represent inefficient use of resources. Solutions are not easy, particularly as the optimal unplanned admission rate or LOS is unclear; although it is tempting to assume that lower admission rates and LOS are desirable, it may be that units with very low admission rates or LOS fail their patients if they are denying care to those who would benefit from inpatient treatment or discharging patients inappropriately early, which might result in higher readmission rates downstream $[45,46]$.

The key question of what can be done to reduce practice variation in potentially avoidable unplanned admissions and LOS is unlikely be answered using observational data however our results alongside the plethora of interventions tested in the wider literature [11] provide some insight. Self-management programmes have been shown to reduce unplanned admissions for patients with COPD [47] and asthma [48] while greater continuity of care with a family physician has been reported to reduce admissions rates [49]. In secondary care, early review by senior clinicians in emergency departments could be effective [50] while the association between increased local bed supply and increased admission rates [34, 23, 24] hints at supply sensitive care [13]. Closer integration between health and social care has shown promising results [51] and has been highlighted as a key aim of the UK government with the introduction of the Better Care Fund [52]. Other interventions such as telemedicine or case management have shown limited or mixed results [11]. Meanwhile, those aiming to curb unnecessary high LOS could introduce secondary care quality improvement initiatives such as clinical pathways $[40,41]$ or guidelines [39, 28, 27].

]The causes of variation specified by authors also serve to highlight the inherent difficulties with conducting and interpreting studies comparing process measures across several geographical units. Demographic and case-mix differences between units are common problems which require relatively complex statistical models and rich datasets to properly address. Failure to do this, as was the case with several studies included in this review, could lead to biased results. Frustratingly, several studies investigated the effect of case mix variables and, despite finding large associations, still presented unadjusted geographical comparisons [26, 53, 22, 54, 55, 28].

There were wide inconsistencies in the methods used to quantify and report variation. Most studies used graphs, maps or tables without any summary statistic quantifying the extent of variation which does not allow easy comparison between studies. Where summary measures were reported they were often statistically naïve and subject to extreme sampling variability, for example the range and coefficient of variation.

\section{Unanswered research questions and future research}

For future empirical work on variations in ACSC admissions and length of stay to be most useful for policy makers it should fulfil a number of criteria: 1) Compare a large number of regions/units to explore the factors associated with admissions and LOS; 2) Adjust for demography and case mix (prevalence/severity) and acknowledge the potential for unmeasured covariates to introduce bias; 3) Explore other, potentially modifiable, causes of variation (e.g. primary care quality, discharge planning) which might inform policy; 4) Provide a summary measure of variation (e.g. SCV) which delineates random and systematic components of variation; 5) Link to outcomes (e.g. inpatient or 90 day mortality) to make sure that the focus remains on patient outcomes rather than just the cost of the process (fewer admissions, shorter LOS).

\section{Conclusion}

Geographic variation in unplanned ACSCs admission rates and LOS is commonplace in high income countries. These large regional differences should be of concern to policymakers particularly as admissions for these conditions are increasing and are potentially avoidable. Interventions to improve the quality of care and curb variations in practice that cannot be 
explained by clinical need are urgently needed. Better empirical work quantifying the extent of unexplained variation in ACSCs admission rates and LOS and exploring factors associated more efficient care and better patient outcomes is needed to help design these interventions.

\section{Additional file}

Additional file 1: Appendix 1. Electronic Search Strategy. Appendix 2. List of Included Ambulatory Care Sensitive Conditions. Appendix 3. Study Quality, Admission Rates. Appendix 4. Study Quality, LOS Appendix 5. Causes of Variation, Admission Rates. Appendix 6. Causes of Variation, Length of Stay. (DOCX $28 \mathrm{~kb}$ )

\section{Abbreviations}

ACSC: ambulatory care sensitive condition; LOS: length of stay; PC: primary care; SC: secondary care; COPD: chronic obstructive pulmonary disease; IQR: inter-quartile range; ICC: inter-class correlation coefficient; COV: coefficient of variation; SCV: systematic component of variation.

\section{Competing interests}

The authors declare that they have no competing interests.

\section{Authors' contributions}

All authors conceived the study and designed the search strategy. JB conducted the search, extracted the data and drafted the manuscript. WH and SP critically revised the article for intellectual content. All authors read and approved the final manuscript.

\section{Acknowledgements}

The authors would like to thank Margaret Burke for help in formulating the electronic search strategy and Charlotte Chamberlain for screening a sample of abstracts.

\section{Author details}

'School of Social and Community Medicine, University of Bristol, Room 2.07, Canynge Hall, 39 Whatley Road, Bristol BS8 2PS, UK. ${ }^{2}$ Professor of Primary Care, School of Social and Community Medicine, University of Bristol, Bristol, UK. ${ }^{3}$ Professor of Health Economics, School of Social and Community Medicine, University of Bristol, Bristol, UK.

\section{Received: 22 January 2015 Accepted: 16 July 2015}

Published online: 13 August 2015

\section{References}

1. Emergency admissions to hospital: managing the demand. Department of Health;2013.

2. Kocher KE, Dimick JB, Nallamothu BK. Changes in the source of unscheduled hospitalizations in the United States. Medical care. 2013;51(8):689-98. doi:10.1097/MLR.0b013e3182992c7b.

3. Dr. Foster Hospital Guide 2012. Dr. Foster Intelligence;2012.

4. Blunt I, Bardsley M, Dixon J. Trends in emergency admissions in England 2004-2009: is greater efficiency breeding inefficiency? London: The Nuffield Trust; 2010 .

5. Graves N, Weinhold D, Tong E, Birrell F, Doidge S, Ramritu P, et al. Effect of healthcare-acquired infection on length of hospital stay and cost. Infection control and hospital epidemiology : the official journal of the Society of Hospital Epidemiologists of America. 2007;28(3):280-92. doi:10.1086/512642.

6. Victor CR, Healy J, Thomas A, Seargeant J. Older patients and delayed discharge from hospital. Health \& Social Care in the Community. 2000;8(6):443-52. doi:10.1046/j.1365-2524.2000.00270.x.

7. Costa AP, Poss JW, Peirce T, Hirdes JP. Acute care inpatients with long-term delayed-discharge: evidence from a Canadian health region. BMC Health Services Research. 2012;12. doi:10.1186/1472-6963-12-172.
8. Glasby J, Littlechild R, Pryce K. Show Me the Way to go Home: A Narrative Review of the Literature on Delayed Hospital Discharges and Older People. British Journal of Social Work. 2004;34(8):1189-97. doi:10.1093/bjsw/bch136.

9. Data Briefing: Emergency hospital admissions for ambulatory care-sensitive conditions: identifying the potential for reductions. The Kings Fund;2012.

10. Freund T, Campbell SM, Geissler S, Kunz CU, Mahler C, Peters-Klimm F, et al. Strategies for Reducing Potentially Avoidable Hospitalizations for Ambulatory Care-Sensitive Conditions. The Annals of Family Medicine. 2013;11(4):363-70. doi:10.1370/afm.1498.

11. Purdy S. Avoiding hospital admissions: What does the research evidence say?, The Kings Fund;2010.

12. Billings J, Zeitel L, Lukomnik J, Carey TS, Blank AE, Newman L. Impact of socioeconomic status on hospital use in New York City. Health affairs (Project Hope). 1993;12(1):162-73.

13. Fisher $\mathrm{ES}$, Wennberg JE. Health care quality, geographic variations, and the challenge of supply-sensitive care. Perspectives in biology and medicine. 2003;46(1):69-79. doi:10.1353/pbm.2003.0004.

14. Purdy S, Griffin T, Salisbury C, Sharp D. Ambulatory care sensitive conditions: terminology and disease coding need to be more specific to aid policy makers and clinicians. Public Health. 2009;123(2):169-73. doi:10.1016/ j.puhe.2008.11.001.

15. Wells G, Shea B, O'Connell D, Peterson J, Welch V, Losos M, Tugwell P. The Newcastle-Ottawa Scale (NOS) for assessing the quality of nonrandomised studies in meta-analyses. 2014.

16. Herzog R, Alvarez-Pasquin MJ, Diaz C, Del Barrio JL, Estrada JM, Gil A Newcastle-Ottawa Scale adapted for cross-sectional studies. 2013.

17. Viera AJ, Garrett JM. Understanding interobserver agreement: the kappa statistic. Family medicine. 2005;37(5):360-3.

18. Landis JR, Koch GG. The measurement of observer agreement for categorical data. Biometrics. 1977;33(1):159-74.

19. Chen J, Normand SL, Wang Y, Krumholz HM. National and regional trends in heart failure hospitalization and mortality rates for Medicare beneficiaries, 1998-2008. JAMA. 2011;306(15):1669-78.

20. Downing A, Rudge G, Cheng Y, Tu YK, Keen J, Gilthorpe MS. Do the UK government's new Quality and Outcomes Framework (QOF) scores adequately measure primary care performance? A cross-sectional survey of routine healthcare data. BMC Health Services Research. 2007;7:166.

21. Klausen HH, Petersen J, Lindhardt T, Bandholm T, Hendriksen C, Kehlet H, et al. Outcomes in elderly Danish citizens admitted with community-acquired pneumonia. Regional differencties, in a public healthcare system. Respiratory Medicine. 2012;106(12):1778-87.

22. Giuffrida A, Gravelle H, Roland M. Measuring quality of care with routine data: avoiding confusion between performance indicators and health outcomes. British Medical Journal. 1999;319(7202):94-8.

23. Morris RD, Munasinghe RL. Geographic Variability In-Hospital Admission Rates For Respiratory-Disease Among The Elderly In The United-States. Chest. 1994;106(4):1172-81. doi:10.1378/chest.106.4.1172.

24. To T, Feldman W, Young W, Maloney SL. Hospitalization rates of children with gastroenteritis in Ontario. Canadian Journal of Public Health. 1996; Revue Canadienne de Sante Publique. 87 (1):62-5.

25. Jin Y, Marrie TJ, Carriere KC, Predy G, Houston C, Ness K, et al. Variation in management of community-acquired pneumonia requiring admission to Alberta, Canada hospitals. Epidemiology and Infection. 2003;130(1):41-51.

26. Barnett RM. Laurence. Practice and ethnic variations in avoidable hospital admission rates in Christchurch, New Zealand. Health \& Place. 2010;16(2):199-208. doi:10.1016/.jhealthplace.2009.09.010.

27. Price LC, Lowe D, Hosker HS, Anstey K, Pearson MG, Roberts CM, et al. UK National COPD Audit 2003: Impact of hospital resources and organisation of care on patient outcome following admission for acute COPD exacerbation. Thorax. 2006;61(10):837-42.

28. Conway PH, Keren R. Factors associated with variability in outcomes for children hospitalized with urinary tract infection. Journal of Pediatrics. 2009;154(6):789-96.

29. Wennberg JE, Barnes BA, Zubkoff M. Professional uncertainty and the problem of supplier-induced demand. Social Science \& Medicine. 1982;16(7):811-24. doi:10.1016/0277-9536(82)90234-9.

30. Project DA. The Dartmouth Atlas of Health Care. 2014. http:// www.dartmouthatlas.org/. Accessed 30/01/2014 2014.

31. NHS Atlas of Variation in Healthcare for People with Diabetes. RightCare; 2012.

32. NHS Atlas of Variation in Diagnostic Services. RightCare; 2013. 
33. Bernal-Delgado E, García-Armesto S, Peiró S. Atlas of Variations in Medical Practice in Spain: The Spanish National Health Service under scrutiny. Health Policy. 2014;114(1):15-30. doi:10.1016/j.healthpol.2013.07.013.

34. Purdy S, Griffin T, Salisbury C, Sharp D. Emergency respiratory admissions: influence of practice, population and hospital factors. Journal of Health Services Research \& Policy. 2011;16(3):133-40. doi:10.1258/jhsrp.2010.010013.

35. Bottle A, Gnani S, Saxena S, Aylin P, Mainous III A, Majeed A. Association Between Quality of Primary Care and Hospitalization for Coronary Heart Disease in England: National Cross-sectional Study. Journal of General Internal Medicine. 2008;23(2):135-41. doi:10.1007/s11606-007-0390-2.

36. Soljak M, Calderon-Larranaga A, Sharma P, Cecil E, Bell D, Abi-Aad G, et al. Does higher quality primary health care reduce stroke admissions? A national cross-sectional study. The British journal of general practice : the journal of the Royal College of General Practitioners. 2011;61(593):e801-7. doi:10.3399/bjgp11X613142.

37. Kiran T, Hutchings A, Dhalla IA, Furlong C, Jacobson B. The association between quality of primary care, deprivation and cardiovascular outcomes: a crosssectional study using data from the UK Quality and Outcomes Framework. Journal of Epidemiology and Community Health. 2010;64(10):927-34. doi:10.1136/jech.2009.098806.

38. Harrison MJ, Dusheiko M, Sutton M, Gravelle H, Doran T, Roland M. Effect of a national primary care pay for performance scheme on emergency hospital admissions for ambulatory care sensitive conditions: controlled longitudinal study. BMJ (Clinical research ed). 2014;349:g6423. doi:10.1136/bmj.g6423.

39. Bahtsevani C, Uden G, Willman A. Outcomes of evidence-based clinical practice guidelines: a systematic review. International journal of technology assessment in health care. 2004;20(4):427-33.

40. Rotter T, Kugler J, Koch R, Gothe H, Twork S, van Oostrum J, et al. A systematic review and meta-analysis of the effects of clinical pathways on length of stay, hospital costs and patient outcomes. BMC Health Services Research. 2008;8(1):265

41. Lemmens KMM, Nieboer AP, Huijsman R. A systematic review of integrated use of disease-management interventions in asthma and COPD. Respiratory Medicine. 2009;103(5):670-91. doi:10.1016/j.rmed.2008.11.017.

42. Pittet $D$, Tarara D, Wenzel RP. Nosocomial bloodstream infection in critically iii patients: Excess length of stay, extra costs, and attributable mortality. JAMA. 1994;271(20):1598-601. doi:10.1001/jama.1994.03510440058033.

43. Kossovsky MP, Sarasin FP, Chopard P, Louis-Simonet M, Sigaud P, Perneger $T V$, et al. Relationship between hospital length of stay and quality of care in patients with congestive heart failure. Quality and Safety in Health Care. 2002:11(3):219-23. doi:10.1136/qhc.11.3.219.

44. Clarke A. Length of in-hospital stay and its relationship to quality of care. Quality and Safety in Health Care. 2002;11(3):209-10. doi:10.1136/qhc.11.3.209

45. Bryan K. Policies for reducing delayed discharge from hospital. British Medical Bulletin. 2010;95(1):33-46. doi:10.1093/bmb/ldq020.

46. Kaboli PJ, Go JT, Hockenberry J, Glasgow JM, Johnson SR, Rosenthal GE, et al. Associations Between Reduced Hospital Length of Stay and 30Day Readmission Rate and Mortality: 14-Year Experience in 129 Veterans Affairs Hospitals. Annals of Internal Medicine. 2012;157(12):837-45. doi:10.7326/0003-4819-157-12-201212180-00003.

47. Zwerink M, Brusse-Keizer M, van der Valk PD, Zielhuis Gerhard A Monninkhof Evelyn M, van der Palen J, et al. Self management for patients with chronic obstructive pulmonary disease. Cochrane Database of Systematic Reviews. 2014. doi:10.1002/14651858.CD002990.pub3.

48. Tapp S, Lasserson TJ, Rowe B. Education interventions for adults who attend the emergency room for acute asthma. The Cochrane database of systematic reviews. 2007;3:Cd003000. doi:10.1002/14651858.CD003000.pub2.

49. Huntley A, Lasserson D, Wye L, Morris R, Checkland K, England H et al. Which features of primary care affect unscheduled secondary care use? A systematic review. BMJ Open. 2014;4 (5). doi:10.1136/bmjopen-2013-004746.

50. White AL, Armstrong PAR, Thakore S. Impact of senior clinical review on patient disposition from the emergency department. Emergency Medicine Journal. 2010;27(4):262-5. doi:10.1136/emj.2009.077842.

51. Curry NH, C. Clinical and service integration, The Kings Fund; 2010

52. England NHS. Better Care Fund Planning. 2014.

53. Ellison-Loschmann L, King R, Pearce N. Regional variations in asthma hospitalisations among Maori and non-Maori. New Zealand Medical Journal. 2004;117 (1188).

54. Starr JM, Thomas B, Whalley LJ. Population risk factors for hospitalization for stroke in Scotland. International Journal of Epidemiology. 1996;25(2):276-81.
55. Claeys MJ, Sinnaeve PR, Convens C, Dubois P, Boland J, Vranckx P, et al. Inter-hospital variation in length of hospital stay after ST-elevation myocardial infarction: results from the Belgian STEMI registry. Acta Cardiologica. 2013;68(3):235-9. doi:10.2143/ac.68.3.2983416.

56. Ansari Z, Ackland MJ, Carson NJ, Choi BCK. Small area analysis of diabetes complications: Opportunities for targeting public health and health services interventions. Australian Journal of Primary Health. 2005;11(3):72-8.

57. Tennant M, Namjoshi D, Silva D, Codde J. Oral health and hospitalization in Western Australian children. Australian Dental Journal. 2000;45(3):204-7.

58. Crighton EJ, Elliott SJ, Moineddin R, Kanaroglou P, Upshur RE. An exploratory spatial analysis of pneumonia and influenza hospitalizations in Ontario by age and gender. Epidemiology \& Infection. 2007;135(2):253-61.

59. Crighton EJ, Elliott SJ, Kanaroglou P, Moineddin R, Upshur RE. Spatiotemporal analysis of pneumonia and influenza hospitalizations in Ontario, Canada. Geospatial Health. 2008;2(2):191-202.

60. Curtis JR, To T, Muirhead S, Cummings E, Daneman D. Recent trends in hospitalization for diabetic ketoacidosis in Ontario children. Diabetes Care. 2002;25(9):1591-6.

61. Bandaranayake D, Jacobs M, Baker M, Hunt D, Wood T, Bissielo A et al. The second wave of 2009 pandemic influenza a (H1N1) in New Zealand, January-October 2010. Eurosurveillance. 2011;16 (6).

62. Dharmalingam A, Pool I, Baxendine S, Sceats J. Trends and patterns of avoidable hospitalisations in New Zealand: 1980-1997. New Zealand Medical Journal. 2004;117 (1198).

63. Magan P, Otero A, Alberquilla A, Ribera JM. Geographic variations in avoidable hospitalizations in the elderly, in a health system with universal coverage. BMC Health Services Research. 2008;8:42.

64. Adams WL, Yuan Z, Barboriak JJ, Rimm AA. Alcohol-related hospitalizations of elderly people. Prevalence and geographic variation in the United States. [Erratum appears in JAMA 1993 Nov 3;270 (17):2055]. JAMA. 1993;270(10):1222-5.

65. Casper M, Nwaise I, Croft JB, Hong Y, Fang J, Greer S. Geographic Disparities in Heart Failure Hospitalization Rates Among Medicare Beneficiaries. Journal of the American College of Cardiology. 2010;55(4):294-9. doi:10.1016/j.jacc.2009.10.021.

66. Gorton CP, Jones JL. Wide geographic variation between Pennsylvania counties in the population rates of hospital admissions for pneumonia among children with and without comorbid chronic conditions. Pediatrics. 2006;117(2):176-80.

67. Holt JB, Zhang X, Presley-Cantrell L, Croft JB. Geographic disparities in chronic obstructive pulmonary disease (COPD) hospitalization among Medicare beneficiaries in the United States. International Journal of Copd. 2011:6(1):321-8.

68. Laditka SB, Laditka JN. Geographic variation in preventable hospitalization of older women and men: Implications for access to primary health care. Journal of Women \& Aging. 1999;11(4):43-56. doi:10.1300/J074v11n04_04.

69. Lanska DJ, Kryscio R. Geographic-Distribution Of Hospitalization Rates, CaseFatality, And Mortality From Stroke In The United-States. Neurology. 1994;44(8):1541-50.

70. Ogunniyi MO, Holt JB, Croft JB, Nwaise IA, Okafor HE, Sawyer DB, et al. Geographic variations in heart failure hospitalizations among medicare beneficiaries in the Tennessee catchment area. American Journal of the Medical Sciences. 2012;343(1):71-7.

71. Feagan BG, Marrie TJ, Lau CY, Wheeler SL, Wong CJ, Vandervoort MK. Treatment and outcomes of community-acquired pneumonia at Canadian hospitals. CMAJ Canadian Medical Association Journal. 2000;162(10):1415-20.

72. Cabre M, Bolivar I, Pera G, Pallares R. Pneumonia Study Collaborative G. Factors influencing length of hospital stay in community-acquired pneumonia: a study in 27 community hospitals. Epidemiology \& Infection. 2004;132(5):821-9.

73. Garau JB F, Perez-Trallero E, Perez JL, Martin-Sanchez AM, Garcia-Rey C, Martin-Herrero JE, et al. Nacer Grp. Factors impacting on length of stay and mortality of community-acquired pneumonia. Clinical Microbiology and Infection. 2008;14(4):322-9. doi:10.1111/j.1469-0691.2007.01915.x.

74. Pozo-Rodriquez F, Lopez-Campos JL, varez-Martinez CJ, Castro-Acosta A, Aguero R, Hueto J et al. Clinical Audit of COPD Patients Requiring Hospital Admissions in Spain: AUDIPOC Study. PLoS ONE [Electronic Resource]. 2012;7 (7).

75. Hosker H, Anstey K, Lowe D, Pearson M, Roberts CM. Variability in the organisation and management of hospital care for COPD exacerbations in the UK. Respiratory Medicine. 2007;101(4):754-61. doi:10.1016/j.rmed.2006.08.016.

76. Roberts CM, Lowe D, Bucknall CE, Ryland I, Kelly Y, Pearson MG. Clinical audit indicators of outcome following admission to hospital with acute 
exacerbation of chronic obstructive pulmonary disease. Thorax. 2002:57(2):137-41.

77. Rudd AG, Irwin P, Rutledge Z, Lowe D, Wade DT, Pearson M. Regional variations in stroke care in England, Wales and Northern Ireland: results from the National Sentinel Audit of Stroke. Royal College of Physicians Intercollegiate Stroke Working Party. Clinical Rehabilitation. 2001;15(5):562-72.

78. Brogan TV, Hall M, Williams DJ, Neuman MI, Grijalva CG, Farris RWD, et al. Variability in processes of care and outcomes among children hospitalized with community-acquired pneumonia. Pediatric Infectious Disease Journal. 2012;31(10):1036-41.

79. Drye EE, Normand SL, Wang Y, Ross JS, Schreiner GC, Han L, et al. Comparison of hospital risk-standardized mortality rates calculated by using in-hospital and 30-day models: an observational study with implications for hospital profiling. [Erratum appears in Ann Intern Med. 2012 Mar 6;156 (5):404]. Annals of Internal Medicine. 2012;156(1 Pt 1):19-26.

80. Krumholz HM, Chen YT, Bradford WD, Cerese J. Variations in and correlates of length of stay in academic hospitals among patients with heart failure resulting from systolic dysfunction. American Journal of Managed Care. 1999:5(6):715-23.

\section{Submit your next manuscript to BioMed Central and take full advantage of:}

- Convenient online submission

- Thorough peer review

- No space constraints or color figure charges

- Immediate publication on acceptance

- Inclusion in PubMed, CAS, Scopus and Google Scholar

- Research which is freely available for redistribution 